\title{
Decision analysis to estimate cost effectiveness in neurosurgery
}

\author{
Sherman C. Stein, M.D., and Mark G. Burnett, M.D. \\ Department of Neurosurgery, Hospital of the University of Pennsylvania, University of Pennsylvania \\ Medical Center, Philadelphia, Pennsylvania
}

\begin{abstract}
Medical decisions often depend, in part, on cost-effectiveness concerns. Decision analysis is frequently used to help resolve these questions. Unfortunately, this technique has received little attention in neurosurgery. Using an example of moderate head injury, the authors illustrate the utility of this powerful tool in estimating the cost effectiveness of neurosurgical management options.
\end{abstract}

\author{
KEY WoRDS - decision support technique - decision analysis - cost effectiveness • \\ computerized tomography $\bullet$ head injury
}

Decision analysis, originally introduced as an economic tool, has been used as an adjunct in medical decision making since the $1970 \mathrm{~s} .{ }^{27,39}$ The technique is especially effective when considerations of cost are among the determining factors in a medical choice. ${ }^{28,32}$

In neurosurgery decision analysis has been very useful for example, in comparing management strategies in cases of unruptured intracranial arteriovenous malformations. In 1989, Fisher ${ }^{12}$ expanded on earlier predictive models. In his article, entitled "Decision analysis: a tool of the future: an application to unruptured arteriovenous malformations," he compared surgery with the natural history of the disease in a hypothetical 25-year-old man. In subsequent publications by him $^{13}$ and others ${ }^{4,18}$ this theme became increasingly sophisticated and applicable.

The demand for evidence-based practice guidelines, increased government regulation, and limitations imposed by managed care have put pressure on those involved in the medical profession to choose among the many management strategies available for a given illness in a given patient. Financial constraints and the desire for optimum use of limited resources have become aspects of treatment decisions. The choices are rarely obvious, and the evidence to support them is often not organized in sufficient detail.

Abbreviations used in this paper: $\mathrm{CT}=$ computerized tomography; ICU = intensive care unit; PRN = as needed.
Decision analysis is being performed with increasing frequency to help simplify difficult medical choices, particularly in the area of cost containment. Publications in the medical literature reflect this remarkable growth. ${ }^{1-3,5,7}$, $9,15,16,19,22,25,26,31,33,34,37,38,44$ Unfortunately, relatively few recent publications have been written on topics of concern to those involved with neurosurgery, ${ }^{17,20,36,41,45}$ and none has dealt with cost. We wrote this article to address a recent cost-effectiveness question and in hopes of rekindling neurosurgery-related interest in this powerful technique.

\section{CLINICAL MATERIAL AND METHODS}

Decision analysis creates a mathematical model that simulates a clinical trial. As most commonly modeled, the "cohort" of patients moves through a sequence of clinical states through Markov modeling, in which a finite series of possible conditions and transitions is defined, specified, and often displayed as a decision tree. The cohort members "transition" from one Markov state to another at discrete intervals. The chain of transitions between states is established using probabilities determined after review of the literature or through indepenent research. Calculated outcomes are based on some numerical value such as cost, quality, and risk-adjusted life years. The process of decision analysis allows for direct comparison of different diagnostic or therapeutic strategies, and it aids in deciding which approach is optimum in a particlar case.

The procedure by which one performs decision analysis 
is best illustrated using an example-in this instance, a question recently addressed to the authors. The Trauma Service at the University of Pennsylvania, as a routine practice, obtains admission head CT scans in all patients who have sustained moderate closed head injury (Glasgow Coma Scale scores 9-13). Those who harbor intracranial lesions either undergo surgery or are observed in the ICU. Over the next 12 to 24 hours (the morning after admission), patients in whom neither deterioration nor recovery to a Glasgow Coma Scale score of 14 or 15 occurs ("gray-zone" patients) undergo a second CT study. The question posed is which of two approaches is more cost effective: 1) to continue to observe these gray-zone patients in the ICU and reserve the second CT study for those who experience clinical deterioration (PRN CT scanning), or 2) to perform routine serial scanning in all, keeping those in whom CT scans have demonstrated deterioration in the ICU and transferring the others to receive less expensive floor care (serial CT scanning).

Software is available to construct decision analyses, perform calculations, and construct the requisite decision trees, graphs, and tables. We approached this relatively simple problem by using only standard computer calculator and drawing programs.

\section{RESULTS}

Data for the primary (baseline case) calculations were obtained from the literature and directly from the hospital billing department. Additional charge-related information was obtained from a 1996 poll conducted by one of the authors (S.C.S.) of five Level 1 trauma centers (Cooper Hospital, Camden, NJ; Johns Hopkins Bayview Medical Center, Baltimore, MD; Loma Linda University Medical Center, Loma Linda, CA; University of Michigan Hospital, Ann Arbor, MI; and Wilford Hall Air Force Hospital, Lackland Air Force Base, TX). In a review of the literature we found a single report in which the author studied short-term outcomes in moderate head injury by assessing routine CT scans. ${ }^{42}$ The author of this study provided adequate data from which to calculate probabilities. These baseline values and the probabilities calculated from them are shown in Table 1.

A number of simplifying assumptions were made. The analysis only covers the day following admission, as detailed day-by-day outcome data are not available for this patient group. No additional fee is added for CT scans obtained in patients in whom deterioration is evidenced, although some hospitals incur greater costs and charge more for urgent and emergency scans. No penalty is assessed for missing a worsening intracranial lesion (such as an accumulating hematoma) until clinical deterioration has occurred.

A decision tree was constructed, incorporating the two management choices, the proportions of patients with resulting outcomes, and the cost of each possible outcome (Fig. 1). The mean per-patient charges can then readily be calculated from the following formulae. For routine serial CT scanning, scans demonstrated stable condition in $58 \%$ and patients can be transferred to floor care (cost: CT scan + floor bed); scans demonstrated worsened condition in $42 \%$ and patients must remain in the ICU (cost: CT scan + ICU bed). For PRN CT scanning, 87\% do well
TABLE 1

Baseline values and probabilities used in decision analysis

\begin{tabular}{lr}
\hline \hline \multicolumn{1}{c}{ Variable } & Value \\
\hline gray-area patients (\%)* & \\
2nd demonstrates worse status than admission CT & $42 \%$ \\
patient requires op \&/or deteriorates after 2nd CT & $13 \%$ \\
2nd CT demonstrates stable or improved status & $58 \%$ \\
patient requires op \&/or deteriorates after 2nd CT & $0 \%$ \\
$\quad$ has demonstrated stable status & \\
hospital charges (\$) $\dagger$ & \\
ICU care & 2152.00 \\
floor care & 1360.00 \\
unenhanced cranial CT scan & 748.00 \\
hospital charges (\$) & \\
ICU care & 1963.17 \\
floor care & 778.67 \\
unenhanced cranial CT scan & 590.60 \\
\hline
\end{tabular}

* Data derived from report published by Stein.

$\dagger$ Data derived from the Hospital of the University of Pennsylvania (2001).

$\ddagger$ Data derived from a five-hospital survey (see Results).

(cost: ICU bed alone) whereas the condition deteriorates in 13\% and patients require urgent CT scanning (cost: CT scan + ICU bed). Actual charges can be substituted into these formulae to calculate relative costs of the two approaches. For the baseline case (Hospital of the University of Pennsylvania, 2001) the mean per-patient cost for a routine serial scan is $(\$ 2108 \times 0.58)+(\$ 2900 \times 0.42)$, or $\$ 2440.64$, and the mean cost for routine ICU care is $(\$ 2152 \times 0.87)+(\$ 2900 \times 0.13)$, or $\$ 2249.24$. Thus, the simple answer to our question is that the use of CT scanning to triage gray-area patients is not cost effective.

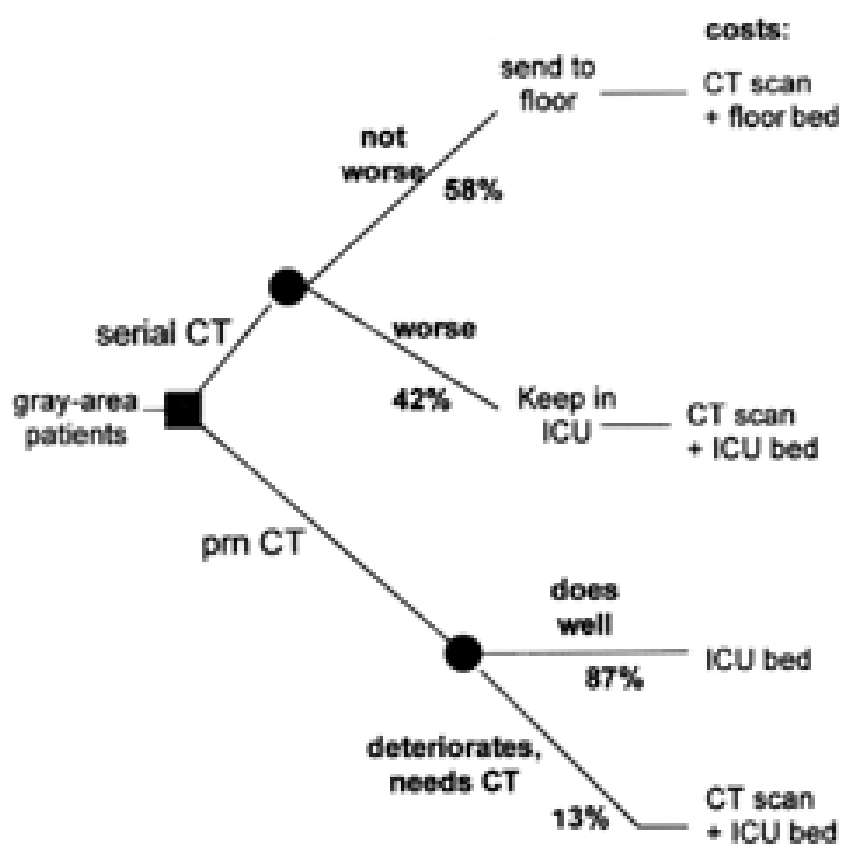

Fig. 1. Decision tree illustrating management choices for "grayarea" patients in clinical example. The various branches represent different decisions or chance outcomes. The percentages denote the calculated probability of each outcome. For each outcome, the components of costs are listed. 


\section{Decision analysis to estimate cost-effectiveness in neurosurgery}

These calculations do not take into account the variability of the data on which they are based. Furthermore, because of the disparate populations from which they are derived, the data are not amenable to reliable statistical analysis. Hence, decision analysis must approach uncertainty another way-that is, through the use of sensitivity analysis. Sensitivity analysis examines the stability of the conclusions when one or more of the underlying parameters are varied by using a wide range of plausible values. In our example, it is intuitively obvious that the total costs are quite sensitive to the relative charges for the three items. If we substitute the values specified in the 1996 survey (Table 1) in the formula, then the cost for for routine serial scanning is only $\$ 1866.76$, somewhat less than the \$2039.95 needed for PRN CT scanning.

The results of sensitivity analysis can be displayed graphically. Figure 2 illustrates the effect of varying the charge for CT scanning in the baseline case. It is clear that this has a much greater effect on the mean per-patient cost of the serial CT option. This is a one-way (one-parameter varied) sensitivity analysis. It can also be observed that, if other charges are constant, serial CT scanning is

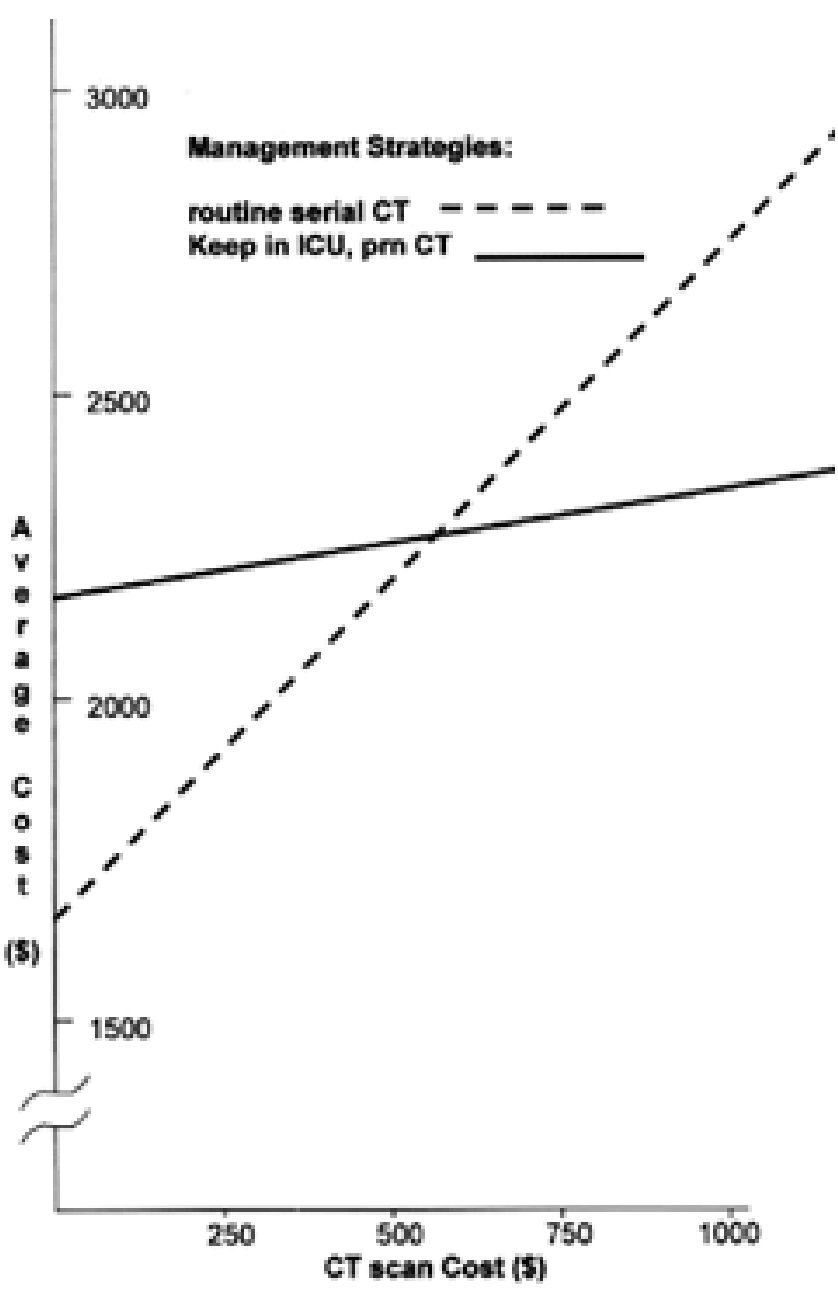

Fig. 2. One-way sensitivity analysis demonstrating the effect of the cost of a CT scan on the comparative costs of two management strategies in the baseline case. the more cost-effective strategy when the charge for a scan is below $\$ 528$.

A two-way sensitivity analysis allows us to vary two parameters. This may permit greater generalization and robustness of the analysis. For example, the graph in Fig. 3 illustrates a two-way sensitivity analysis. Here the daily charges for hospital and ICU beds are expressed as multiples of the charge for a CT scan. Because the financial units have the same base, the model applies equally to relative charges and costs. As is illustrated graphically, whenever the cost (or the charge) of an ICU bed exceeds that of a hospital bed by at least 1.5 times the cost (or charge) of obtaining a CT study, routine serial CT scanning is the more cost-effective strategy.

In three-way sensitivity analysis the robustness of the model is further tested. For example, it could be argued that a delayed deterioration rate of $13 \%$ used in the study is based on a small series of patients and could be inadequate. As is demonstrated in Fig. 4, however, decisions based on the model remain quite stable under much higher or lower rates. Hence, within the plausible range, the delayed deterioration rate has little effect on costs.

\section{DISCUSSION}

As the example demonstrates, it is possible to use decision analysis to resolve questions of cost effectiveness. Not only can the model be used to determine which of the

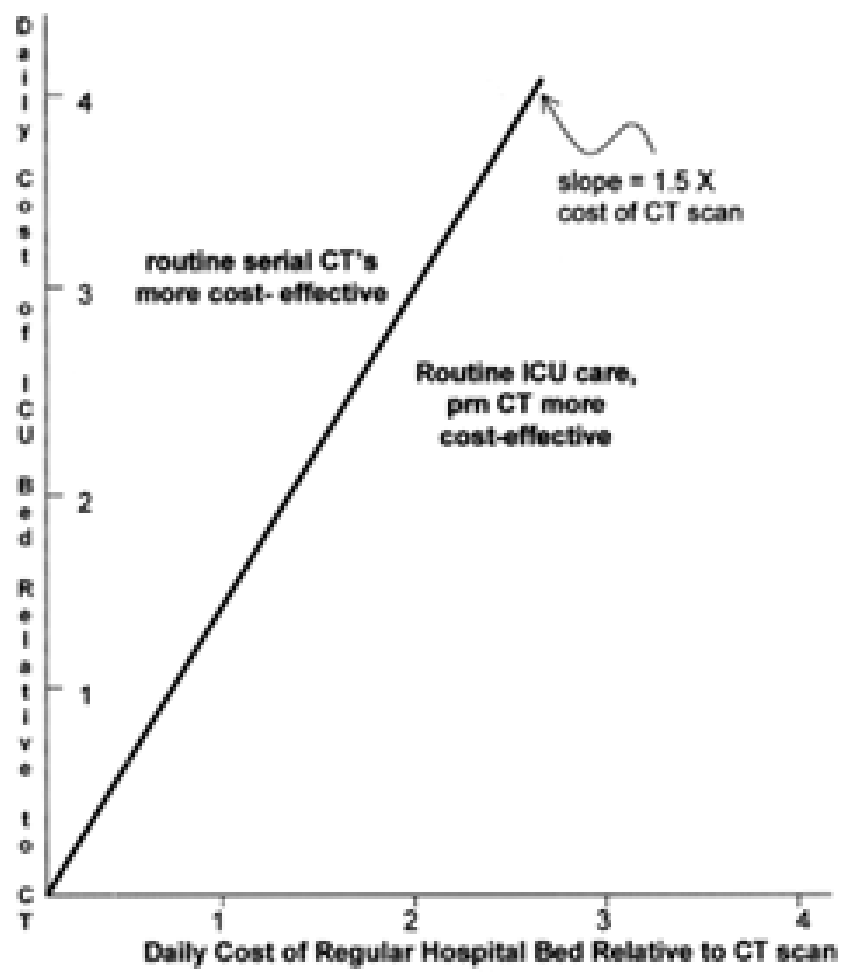

Fig. 3. Two-way sensitivity analysis showing the relationship between costs of bed and CT scan. The plotted line represents the threshold at which the cost effectiveness of the two strategies is the same. This analysis can be applied to any setting in which either costs or charges are known. 


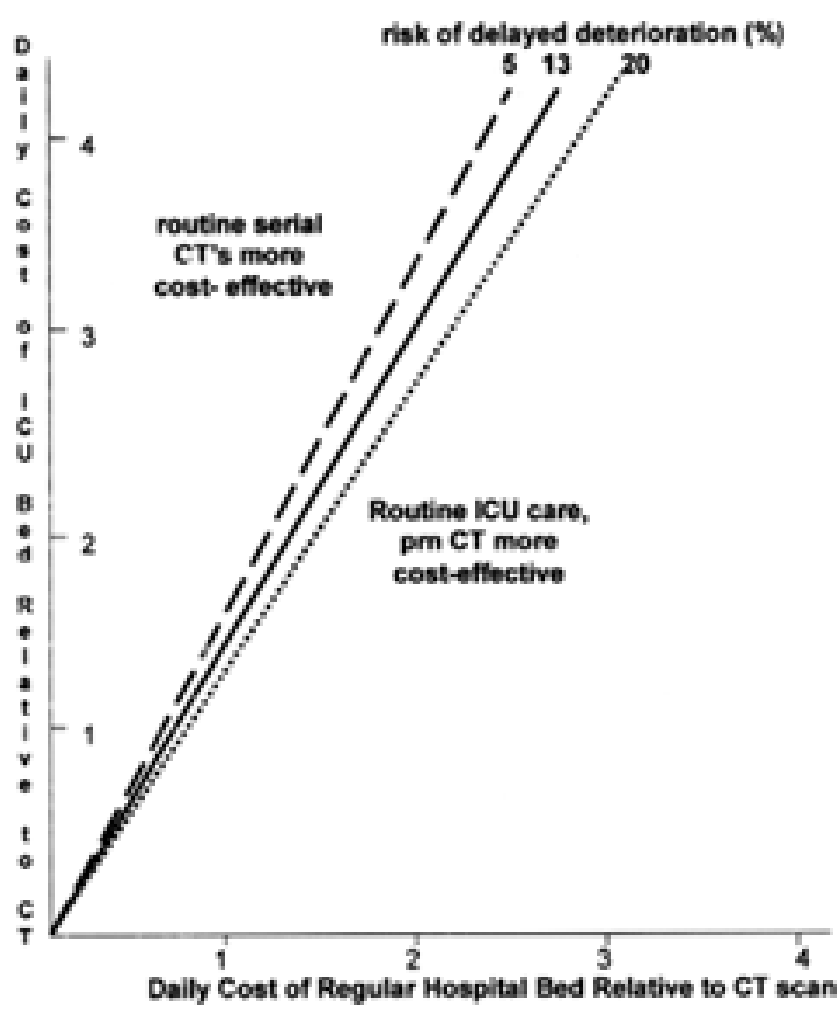

Fig. 4. Three-way sensitivity analysis demonstrating the relationship between costs of bed and CT scan, as well as the effect of varying the rate of delayed deterioration. The three isobars represent the thresholds at which the cost effectiveness of the two strategies are the same. As the risk of delayed deterioration rises, the value of routine CT scanning improves. The effect of varying deterioration rate, however, is small.

two suggested strategies costs less under the specified charges, it can be adapted to make the determination under any combination of charges or costs. The finding that routine serial CT scanning is useful under a particular set of circumstances contradicts conventional thinking in neurosurgical practice. , $8,14,21,23,24,35,40$

It must be stressed that our simplified example may give the impression that decision analysis is a technique easily adapted to all clinical situations. In the example it is assumed that the outcomes of the two management strategies are the same, even though a proportion of patients in whom hematomas are not diagnosed before deterioration has occurred may suffer harm. ${ }^{29}$ Because efficacy often varies among alternative treatment plans, complex calculations are often necessary to compare the "effectiveness" aspect of a cost-effectiveness study. ${ }^{30}$ There are no calculations for costs beyond the 1 day in the scenario. We used charges rather than costs, as the latter are usually difficult to obtain. Of note, the model is equally valid for both. No account is taken of other, possibly important, measures of utility. For example, if the number of ICU beds is especially limited it may be necessary to factor in a premium for freeing up these beds to be occupied by other patients in need. When a clinical decision must take into account factors such as patient preference, multiple iterations involving several sequential choices or time intervals, and outcome measures, including quality- and risk-adjusted life years, calculations may become exceedingly complex.

Additionally, the conclusions obtained using decision analysis are not universally accepted. Critics have contended that there are a number of shortcomings associated with the technique, including its tendencies to define problems too narrowly, to be too removed from clinical situations, and to be too time-consuming and complex. As is the case in other approaches to decision making, it is limited by a lack of high-quality data and a gold standard for utility. Some critics have asserted that decision analysis is used to replace clinical judgment, thus dehumanizing patient care. Its emphasis on the consequences of decisions as the goal, however, has even been questioned. ${ }^{10}$

Nevertheless, decision analysis remains a powerful tool with which to clarify clinical options. The process is systematic, explicit, and transparent. It can be used to attempt to give appropriate weight to both empirical data and patient preferences. The decision analysis process cannot replace a well-designed, well-controlled, multicenter randomized trial or a careful metaanalysis of such trials. It can, however, put the results of trials in a quantitative context so that they can be used in decision making. It can also serve as a surrogate for controlled trials in the many cases in which they have yet to be performed. It can even be used to assist in the planning of well-designed clinical trials. There are several recent discussions of the advantages and disadvantages of decision analysis in the context of patient care. ${ }^{11,43,46}$

Perhaps the characterization offered by Fisher ${ }^{12}$ of decision analysis as "a tool of the future," was a bit optimistic. He was, however, quite correct in pointing out its utility for addressing neurosurgery-related problems. The purpose of this paper is to stress that the benefits of decision analysis include neurosurgical judgments with socioeconomic implications.

\section{References}

1. Allen MW, Hendi P, Schwimmer J, et al: Decision analysis for the cost effectiveness of sestamibi scintimammography in minimizing unnecessary biopsies. Q J Nucl Med 44:168-185, 2000

2. Arguedas MR, Dupont AW, Wilcox CM: Where do ERCP, endoscopic ultrasound, magnetic resonance cholangiopancreatography, and intraoperative cholangiography fit in the management of acute biliary pancreatitis? A decision analysis model. Am J Gastroenterol 96:2892-2899, 2001

3. Arguedas MR, Heudebert GR, Wilcox CM: Surveillance colonoscopy or chemoprevention with COX-2 inhibitors in averagerisk post-polypectomy patients: a decision analysis. Aliment Pharmacol Ther 15:631-638, 2001

4. Auger RG, Wiebers DO: Management of unruptured intracranial arteriovenous malformations: a decision analysis. Neurosurgery 30:561-569, 1992

5. Carlos RC, Bree RL, Abrahamse PH, et al: Cost-effectiveness of saline-assisted hysterosonography and office hysteroscopy in the evaluation of postmenopausal bleeding: a decision analysis. Acad Radiol 8:835-844, 2001

6. Chao A, Pearl J, Perdue P, et al: Utility of routine serial computed tomography for blunt intracranial injury. J Trauma 51: 870-875, 2001

7. Chey WD, Fendrick AM: Noninvasive Helicobacter pylori testing for the "test-and-treat" strategy: a decision analysis to assess the effect of past infection on test choice. Arch Intern Med 161:2129-2132, 2001 
8. Cope DN, Date ES, Mar EY: Serial computerized tomographic evaluations in traumatic head injury. Arch Phys Med Rehabil 69:483-486, 1988

9. Dietlein M, Weber K, Gandjour A, et al: Cost-effectiveness of FDG-PET for the management of solitary pulmonary nodules: a decision analysis based on cost reimbursement in Germany. Eur J Nucl Med 27:1441-1456, 2000

10. Eisinger F: Decision analysis in patients' care. Lancet 358: 2173, 2001

11. Elwyn G, Edwards A, Eccles M, et al: Decision analysis in patient care. Lancet 358:571-574, 2001

12. Fisher WS III: Decision analysis: a tool of the future: an application to unruptured arteriovenous malformations. Neurosurgery 24:129-135, 1989

13. Fisher WS III: Therapy of AVMs: a decision analysis. Clin Neurosurg 42:294-312, 1995

14. French BN, Dublin AB: The value of computerized tomography in the management of 1000 consecutive head injuries. Surg Neurol 7:171-183, 1977

15. George B, Harris A, Mitchell A: Cost-effectiveness analysis and the consistency of decision making: evidence from pharmaceutical reimbursement in Australia (1991 to 1996). Pharmacoeconomics 19:1103-1109, 2001

16. Gracia CR, Brown HA, Barnhart KT: Prophylactic methotrexate after linear salpingostomy: a decision analysis. Fertil Steril 76:1191-1195, 2001

17. Holloway RG, Mushlin AI: Intracranial mass lesions in acquired immunodeficiency syndrome: using decision analysis to determine the effectiveness of stereotactic brain biopsy. Neurology 46:1010-1015, 1996

18. Hudgins WR: Management of unruptured intracranial arteriovenous malformations: a decision analysis. Neurosurgery 33: 172-173, 1993

19. James M, Hunt K, Burr R, et al: A decision analytical cost analysis of offering ECV in a UK district general hospital. BMC Health Serv Res 1:6, 2001

20. Kasner SE, Kimmel SE: Accuracy of initial stroke subtype diagnosis: a decision analysis. Cerebrovasc Dis 10:18-24, 2000

21. Kobayashi S, Nakazawa S, Otsuka T: Clinical value of serial computed tomography with severe head injury. Surg Neurol 20:25-29, 1983

22. Le TP, Miller LG: Empirical therapy for uncomplicated urinary tract infections in an era of increasing antimicrobial resistance: a decision and cost analysis. Clin Infect Dis 33:615-621, 2001

23. Lee TT, Aldana PR, Kirton OC, et al: Follow-up computerized tomography (CT) scans in moderate and severe head injuries: correlation with Glasgow Coma Scores (GCS), and complication rate. Acta Neurochir 139:1042-1048, 1997

24. Macpherson P, Jennett B, Anderson E: CT scanning and surgical treatment of 1551 head injured patients admitted to a regional neurosurgical unit. Clin Radiol 42:85-87, 1990

25. Marshall D, Simpson KN, Earle CC, et al: Economic decision analysis model of screening for lung cancer. Eur J Cancer 37: 1759-1767, 2001

26. McNeil BJ: Changing roles of decision analysis and cost-effectiveness analyses in medicine and radiology. Eur Radiol 10 (Suppl 3):S340-S343, 2000

27. McNeil BJ, Keller E, Adelstein SJ: Primer on certain elements of medical decision making. N Engl J Med 293:211-215, 1975

28. McNeil BJ, Varady PD, Burrows BA, et al: Measures of clinical efficacy. Cost-effectiveness calculations in the diagnosis and treatment of hypertensive renovascular disease. N Engl J Med 293:216-221, 1975
29. Miller JD, Murray LS, Teasdale GM: Development of a traumatic intracranial hematoma after a "minor" head injury. Neurosurgery 27:669-673, 1990

30. Naglie G, Krahn MD, Naimark D, et al: Primer on medical decision analysis: Part 3-Estimating probabilities and utilities. Med Decis Making 17:136-141, 1997

31. Park KC, Schwimmer J, Shepherd JE, et al: Decision analysis for the cost-effective management of recurrent colorectal cancer. Ann Surg 233:310-319, 2001

32. Pauker SG, Kassirer JP: Therapeutic decision making: a costbenefit analysis. N Engl J Med 293:229-234, 1975

33. Piccoli A: Elementary clinical decision analysis in evidencebased nephrology. J Nephrol 13:419-432, 2000

34. Reeves SW, Friedman DS, Fleisher LA, et al: A decision analysis of anesthesia management for cataract surgery. Am J Ophthalmol 132:528-536, 2001

35. Roberson FC, Kishore PR, Miller JD, et al: The value of serial computerized tomography in the management of severe head injury. Surg Neurol 12:161-167, 1979

36. Ruigrok YM, Rinkel GJ, Buskens E, et al: Perimesencephalic hemorrhage and CT angiography: a decision analysis. Stroke 31:2976-2983, 2000

37. Sarasin FP: Decision analysis and its application in the clinical medicine. Eur J Obstet Gynecol Reprod Biol 94:172-179, 2001

38. Scheiman JM, Bandekar RR, Chernew ME, et al: Helicobacter pylori screening for individuals requiring chronic NSAID therapy: a decision analysis. Aliment Pharmacol Ther 15:63-71, 2001

39. Schwartz WB, Gorry GA, Kassirer JP, et al: Decision analysis and clinical judgment. Am J Med 55:459-472, 1973

40. Servadei F, Nanni A, Nasi MT, et al: Evolving brain lesions in the first 12 hours after head injury: analysis of 37 comatose patients. Neurosurgery 37:899-907, 1995

41. Stein SC: Management of minor closed head injury. Neurosurg Q 6:108-115, 1996

42. Stein SC: Outcome from moderate head injury, in Narayan RK, Wilberger JE Jr, Povlishock JT (eds): Neurotrauma. New York: McGraw-Hill, 1996, pp 755-765

43. Tavakoli M, Davies HT, Thomson R: Decision analysis in evidence-based decision making. J Eval Clin Pract 6:111-120, 2000

44. Urbach DR, Khajanchee YS, Jobe BA, et al: Cost-effective management of common bile duct stones: a decision analysis of the use of endoscopic retrograde cholangiopancreatography (ERCP), intraoperative cholangiography, and laparoscopic bile duct exploration. Surg Endosc 15:4-13, 2001

45. Videtic GM, Gaspar LE, Zamorano L, et al: Implant volume as a prognostic variable in brachytherapy decision-making for malignant gliomas stratified by the RTOG recursive partitioning analysis. Int J Radiat Oncol Biol Phys 51:963-968, 2001

46. Wears RL: The aerial palaces of decision analysis redux. Acad Emerg Med 7:380-382, 2000

Manuscript received February 4, 2002.

Accepted in final form March 7, 2002.

Address reprint requests to: Sherman C. Stein, M.D., Department of Neurosurgery, Hospital of the University of Pennsylvania, Fifth Floor Silverstein Building, University of Pennsylvania Medical Center, 3400 Spruce Street, Philadelphia, Pennsylvania 19104. 